\title{
Improvements in Agile Model using Hybrid Theory for Software Development in Software Engineering
}

\author{
Ratnmala R. Raval \\ Computer Department \\ School of Engineering \\ RK University, \\ Rajkot, Gujarat
}

\author{
Haresh M. Rathod \\ Computer Department \\ School of Engineering \\ RK University, \\ Rajkot, Gujarat
}

\begin{abstract}
Agile is a nothing but different way of managing IT development players and software projects. Various Agile methodologies are scrum, xp method, lean programming, and unified process. it is combining the philosophy of various process models and a set of development guidelines. This attitude encourages customer satisfaction, early incremental delivery of software small, highly motivated project teams. Agile development can provide important benefits, but due to some limitations its not applicable to all projects, product, people and all situations. Although Agile methodology is broadly accepted in software development. But it's not suitable for undergraduate and small client based projects.. In this paper, we present new hybrid agile method which combines philosophy of waterfall, iterative waterfall, Prototypr, spiral as well as pure agile. Then we applied it on small client based project and compare Results with other process model. We found some factors which are battered in hybrid agile like cost reduction, time for implementation, good customer satisfaction and clear risk identification.
\end{abstract}

\section{Keywords}

Software process model, agile model, Hybrid agile model.

\section{INTRODUCTION}

Software systems start with and go through a chain of route that report for their beginning, initial development, dynamic operation, keep, and leaving from one production to another. This research sort out and examines a various methods for describing or model how software systems are developed. At the very first level its starts with background and categorization of famous software life cycle models which are given in text books today's cutthroat market and with the need to be highly immediate, the process of developing software systems has grown significantly. The lack of ability of the unadventurous development paradigm to meet the new challenges of fast software delivery and quick change management gave birth to the agile paradigm [2] Agile methodology is widely used in software development. But it's not suitable for undergraduate projects. In This Research we Proved that how agile Methodology is not suitable for Undergraduate client based projects and Present hybrid agile model which is suitable for the same.

\section{SOFTWARE PROCESS MODEL}

A software process model is an abstract representation of Processes which are using to develop the software [2]. It simply follows the SDLC methodology which includes Analysis, Design, Implementation, Testing, and Maintains. There are various process models in software development like waterfall, Iterative Waterfall, incremental, spiral, rad and agile. Waterfall model is dead [2], Iterative and incremental have good theory for development but not executed properly in industries. The main unique feature of the spiral model is that it creates a risk-driven advance to the software process rather than an above all documentdriven or code-driven process. It incorporates many of the strengths of other models and resolves many of their difficulties.

\section{$2.1 \quad$ Agile}

Agile development methodology provides opportunities to assess the direction of a project throughout the development lifecycle. This is achieved through regular cadences of work, known as sprints or iterations, at the end of which teams must present a potentially shippable product increment. By focusing on the repetition of abbreviated work cycles as well as the functional product they yield, agile methodology is described as "iterative" and "incremental." In waterfall, development teams only have one chance to get each aspect of a project right. In an agile paradigm, every aspect of development requirements, design, etc. - is continually revisited throughout the lifecycle. Various Agile methodologies are scrum, xp method, lean programming, and unified process. Agile methods were developed in effort to overcome perceived and actual weakness in conventional software engineering. 


\subsection{Principles of Agile Manifesto [7].}

1. The utmost Priority is to satisfy the customer through early and continues delivery of valuable software.

2. Welcome changing requirements, even late in development. Agile process harness change for customer competitive advantages

3. Deliver working software frequently, from a couple weeks to a couple of months with a shorter time scale.

4. Business people and developer must work together daily throughout the project.

5. Build project around motivated individuals, give them the environment and support they need and trust to get the job done.

6. The most efficient and effective method for conveying information to and within development term is face to face conversation.

7. Working software is primary measure of progress.

8. Agile process promote sustainable development the developer and user should be able to maintain a cost pace identify.

9. Continues attention to technical excellence and good design enhance agility.

10. At regular interval the team reflect how to become more effective and turns to adjust behavior according.

\subsection{Agile adoption pitfalls [7].}

1. Improper planning.

2. Going too fast

3. Insufficient coaching

\subsection{Myths about agile [7].}

1. Agile is fad.

2. Agile isn't disciplined.

3. Agile means we don't plan.

4. Agile means no Documentation.

5. Agile doesn't scale.

6. Agile is unsuitable for regulated environment.

7. Agile means we don't know what will be delivering.

\section{PROPOSED SOLUTION}

As agile methodology is not perfectly suitable for small client based project [10] here we consider medical inventory system working scenario is Maintain medical stock, store vendor detail, company detail, doctor detail. Maintain account like today vouchers, monthly sale, and yearly sale. Utility like backup and restore. Search based on company name, product name. Alert regarding Expire date.

\subsection{Steps for Proposed solution}

1. Apply theory of various process models on medical inventory system.

2. For applying model consider factors choosing the life cycle model

3. Calculate parameters generated after applying the model theory.

4. Apply hybrid agile model theory on system.

5. Compare the result with previous model result

\section{ANALYSIS \& OBSERVATION}

\subsection{Apply waterfall model}

\subsubsection{Analysis}

1. Team member-4

2. Working hour-7

3. Man power cost 150rs/day(based on it survey)

4. Project duration:52 days

5. Cost analysis for man power is 31200

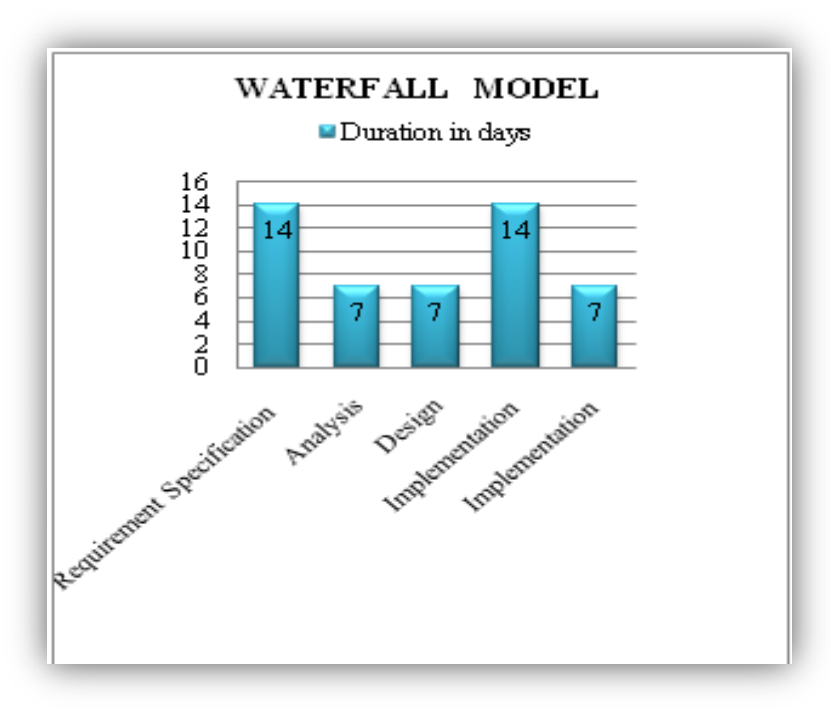

Figure 1. Waterfall model scheduling Chart.

6. Chance to generate risk area -high

7. Customer satisfaction -very low medium

8. Customer feedback-nil

9. Change in requirements will increase cost and time 


\subsection{Apply iterative waterfall model}

\subsubsection{Characteristics}

Same as waterfall, but you can go back to previous phases after executing one face so to go back stage requires more days to complete.

RS-Requirements Specification

\section{A-Analysis \\ D-Design}

\section{I-Implementation}

IT-Implementation \& Test

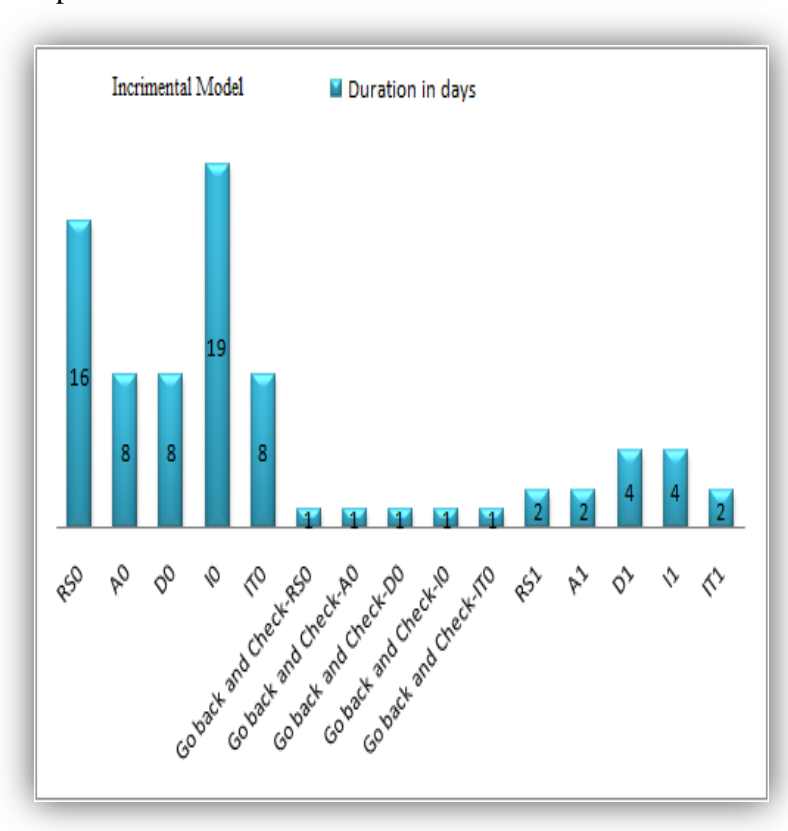

Figure-2 Iterative waterfall model scheduling Chart.

\subsubsection{Analysis}

1. Team member-4

2. Working hour-7

3. Manpower cost per day-150rs/day

4. Project duration -68 days

5. Cost Analysis for man power $-40,800$

6. Chance to generate risk are-Medium (due to go back)

7. Customer Satisfaction is- medium

8. Customer Feedback -nil

9. Change in requirements will increase time and cost.

\subsection{Apply Spiral model}

\subsubsection{Analysis}

1. Team member-4

2. Working hour-7

3. Manpower cost per day-150rs/day
4. Project duration -53 days

5. Cost Analysis for man power $-31,800$

6. Chance to generate risk are -Medium (due to go back)

7. Customer Satisfaction is- Good

8. Customer Feedback -yes

9. Change in requirements will not increase the time and cost much.(Because customer satisfaction and modification phase

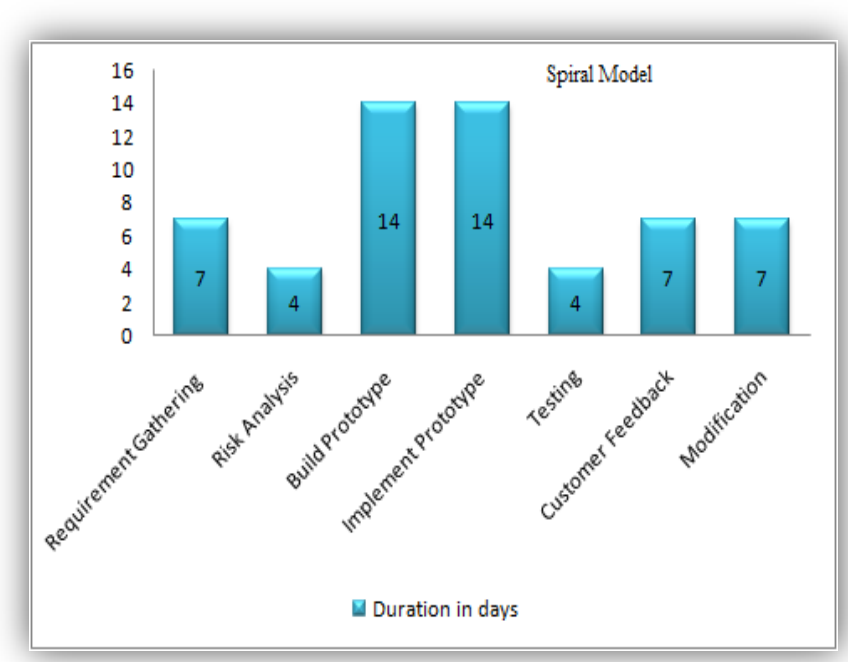

Figure-3 Spiral models scheduling Chart.

\subsection{Apply pure agile model}
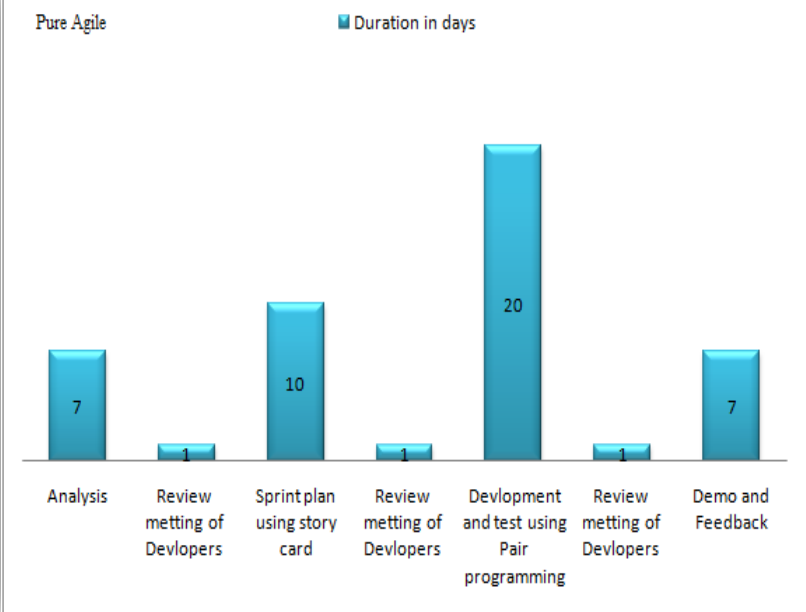

Figure-4 Pure agile model scheduling Chart.

\subsubsection{Analysis}

1. Team member:7(As pure agile team criteria)

2. Working hour:10(Agile believes sprint planning)

3. Man power cost per day:300rs/day(Agile team members are experts and Experienced) 
4. Project duration :47 days

5. Cost Analysis for man power :98,700

6. Chance to generate risk are :very low (due to go back)

7. Customer Satisfaction is: very good

8. Customer Feedback :yes

9. Change in requirements will not increase time and cost much .(because customer satisfaction and modification phase

\subsection{Apply hybrid agile Theory}

\subsubsection{Hybrid agile working}

1. Pure agile team members are more and experts, but in the hybrid agile team member are mixed.

2. Pure agile planning dose using story cards (generally useful when large and complex project) which is more time consuming. Hybrid agile maintain a list of requirements (suitable for the small client based project)

3. Risk driven approach are not single phase in pure agile. Pure agile says frequently communicate with the client so, but the client's point of view and developer's point of view can't be same for the system development, frequent face to face conversation is not much suitable approach for reduce risk so the Hybrid agile theory has one phase of risk analysis. Face to face communication meting is once or two times with customers.

4. In pure agile if requirements are changing (due to defect or some additional requirements generated) after completing the project will defiantly increase cost time and effort, hybrid agile is also taking the concepts of agile maturity model which include change pleas in requirements .

\subsubsection{Analysis}

1. Working hour:10(Agile believes sprint planning)

2. Man power cost per day: 300rs/day (Agile team members are experts and Experienced) +150 Rs/day(basic Developers as per waterfall criteria)

3. Project duration :47 days

4. Cost Analysis for man power :50,400

5. Chance to generate risk are :very low (due to go back)

6. Customer Satisfaction is: very good

7. Customer Feedback :yes

8. Change in requirements will not increase the time and cost much.(Because customer satisfaction and modification phase .

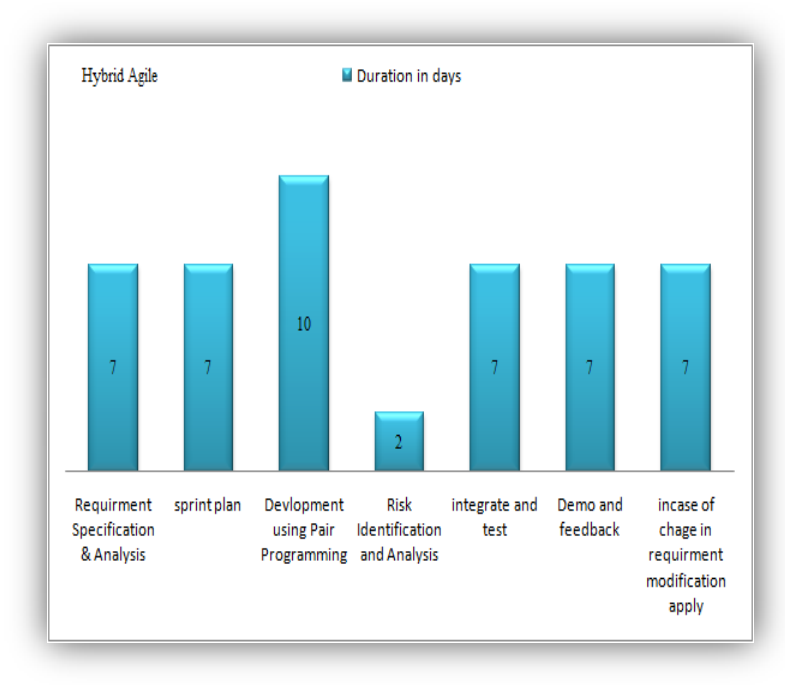

Figure-5 Hybrid model scheduling Chart

\section{CONCLUSION}

After applying different process models theory and hybrid agile theory in medical inventory system. Figure 6 Comparison chart says that hybrid agile is better than previous as well pure agile model. With the help of hybrid theory, we can achieve better customer satisfaction, low prize for development, perfect risk identification, limited complexity and also less time of development. For further research on the agile model in software development researcher should focus on following aspects.

1. In this paper we present new hybrid theory as proposed solution, for further research researcher should apply this theory in various small software industry and find out efficacy of hybrid theory.

2. Implement any small system and apply function point and Cocomo methods for comparison of hybrid theory.

\section{ACKNOWLEDGMENTS}

Thanks to the R.K University Department Of Computer Engineering who have contributed towards development of the Paper. 


\section{EXPERIMENTAL RESULT}

7.1Comparison graph of various process models vs. hybrid agile model

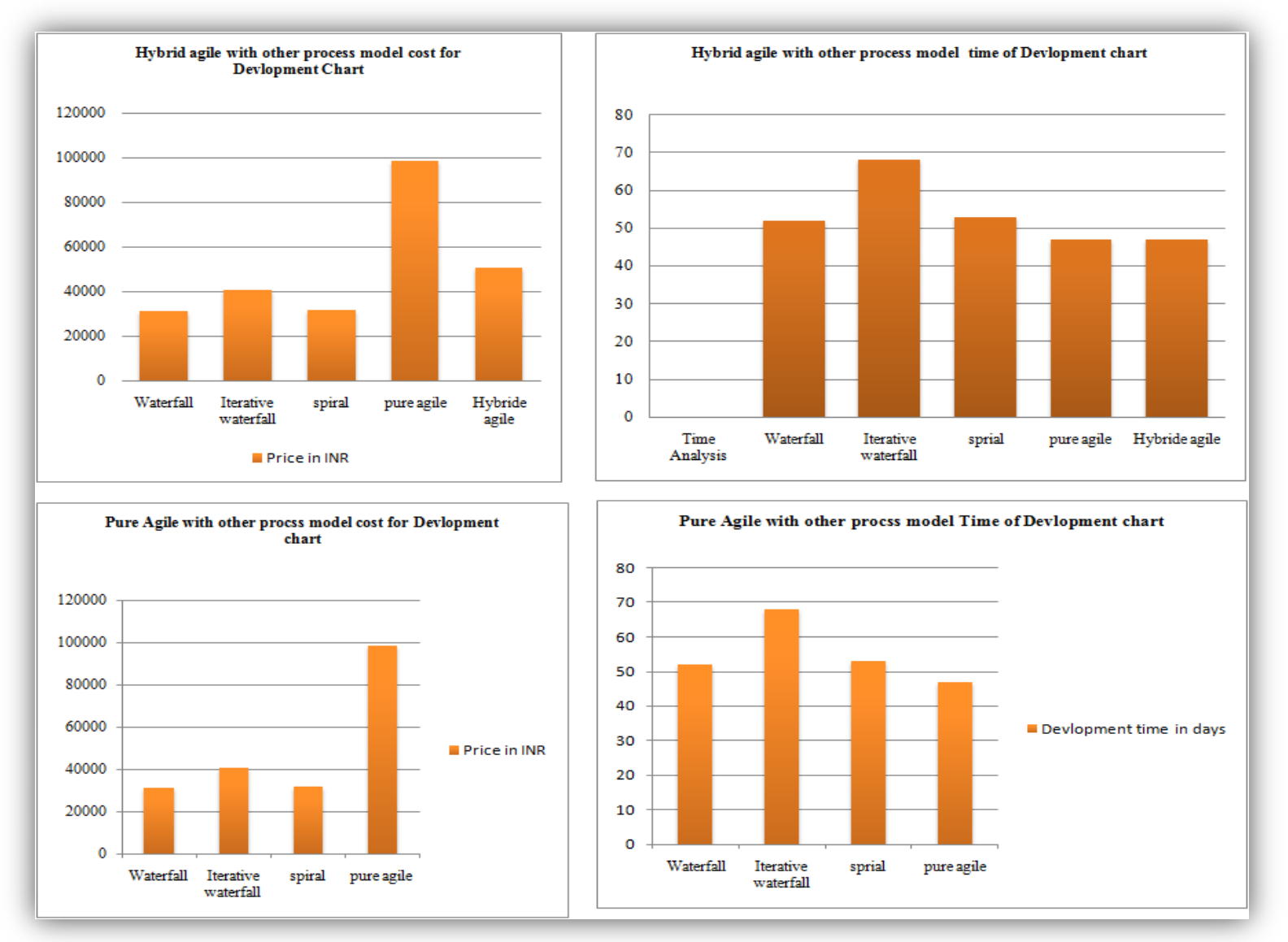

Figure-6 Comparison chart of before hybrid agile \& after hybrid agile

\section{REFERENCES}

[1] Efficiency of Spiral Model by applying Genetic Algorithm 1 Sachin Sharma, 2Anupriya Jain, 3Seema Sharma, 4Sonia Duggal Dept. Of Computer Applications, Manav Rachna International University, Faridabad, Haryana, India. I S S N : 2229 - 4333 ( P r i n t) | I S S N : 0 976 - 8491 (On 1 i n e ) IJCST Vol. 2, Issue 2, June 2011.

[2] A Comparison between Five Models of Software Engineering IJCSI International Journal of Computer Science Issues, Vol. 7, Issue 5, September 2010 ISSN (Online): 1694

[3] What Does Research Say About Agile and Architecture?2010 Fifth International Conference on Software Engineering Advance ,Hongyu Pei Breivold (Industrial Software Systems-ABB Corporate Research) Daniel Sundmark, Peter Wallin and StigLarsson (Mälardalen University) Västerås, Sweden

[4] A meta model-based approach for customizing and assessing agile methods, 2012 Eighth International Conference on the Quality of Information and Communications Technology. Hajer Ayed, Beno^1t Vanderose and Naji Habra PReCISE Research Center University of Namur, Belgium.
[5] Quality Assurance in Agile -A study toward achieving excellence Agile India 2012 Sonali Bhasin Nokia Siemens Networks, Gurgaon, India.

[6] Customizable Agile Software Quality Assurance Model- Information Science and Service Science (NISS), 2011 5th International Conference on New Trends in (Volume:2 )24-26 Oct. 2011.

[7] Improvements for agile manifesto and make agile applicable for undergraduate research projectsM.A.S.C.Manamendra,K.N.Manathunga, K.H.D. Perera and N.Kodagoda Department of Information Technology, Faculty of ComputingSri Lanka Institute of Information Technology - SUIT Colombo, Sri Lanka The 8th International Conference on Computer Science \& Education (ICCSE 2013) April 26-28, 2013. Colombo, Sri Lanka

[8] An Agile Methodology Based Model forChangeOriented Software Engineering Naresh Kumar Nagwani, Pradeep Singh Department of Computer Sc. \& Engg. National Institute of Technology, Raipur International Journal of Recent Trends in Engineering, Vol 1, No. 1, May 2009. 
[9] A Structured Framework for Assessing the "Goodness" of Agile Methods Shvetha Soundararajan and James D. Arthur Department of Computer Science Virginia Tech 2011 18th IEEE International Conference and Workshops on Engineering of Computer-Based Systems

[10] Emerging Trends in Software Engineering presented by Roger S. Pressman, Ph.D.R.S. Pressman \& Associates, Inc. Boca Raton, Florida USA January, 2009
[11] A Methodology for Assessing Agile Software Development MethodsShvetha Soundararajan, James D. Arthur and Osman Balci Department of Computer ScienceVirginia Tech Blacksburg, 2012 Agile Conference.

[12] Enabling Agile Testing Through Continuous Integration-2009 Agile Conference Sean Stolberg Pacific Northwest National Laboratory. 